\title{
Effect of Scribes on Efficiency in Academic Ophthalmology Practice
}

\author{
Meredith Furst, BS ${ }^{1}$ Edward Chu, MD ${ }^{1}$ Kendall Wannamaker, MD ${ }^{1}$ Brian Planchard, MD ${ }^{1}$ \\ Lisa Pacheco, MA ${ }^{1}$ Laura Vigil, $\mathrm{COA}^{1}$ Tarana Ahmed, $\mathrm{COA}^{1}$ Daniel Johnson, MD ${ }^{1}$ \\ Corey Waldman, $\mathrm{MD}^{1}{ }^{10}$
}

${ }^{1}$ Department of Ophthalmology, UT Health San Antonio, San Antonio, Texas

Address for correspondence Corey Waldman, MD, Department of Ophthalmology, UT Health San Antonio, 7703 Floyd Curl Drive, MSC J Acad Ophthalmol 2020;12:e273-e276. 6230, San Antonio, TX 78229 (e-mail: WaldmanC@uthscsa.edu).

\begin{abstract}
Objective The aim of the study is to determine the effects of scribes on efficiency in an academic ophthalmology practice.

Design This is a quality improvement study conducted by two ophthalmologists at an academic ophthalmology practice at UT Health San Antonio from January 2018 to April 2018. Implementation of scribes in practice was the primary intervention. Session time, patient encounter time, and template time adherence were recorded pre- and post-intervention. A second retrospective arm of the study at the same institution was performed to evaluate long-term effects of scribes on efficiency in ophthalmology practice on session times and patient volume 12 to 18 months after intervention.

Main Outcome Measures Primary study outcomes and measures were the effect of scribes in academic ophthalmology practice on physician efficiency in terms of clinic session time, individual encounter time, and amount of patients seen per session, in addition to time adherence based on type of patient encounter.

Results Eighty-three patients and 17 half-day clinic sessions and 169 patients and 21 half-day clinic sessions were included in the preintervention and post-intervention datasets, respectively. Number of patients per session was approximately 15 and was kept similar pre- and post-intervention $(p=0.45)$. Mean preintervention session time was $265.0 \pm 31.4$ minutes, in contrast to $223.4 \pm 19.9$ minutes after intervention $(p<0.001)$. Mean preintervention patient encounter time was $15.0 \pm 8.3$ minutes, while the mean encounter time after intervention was $10.9 \pm 7.0$ minutes $(p<0.005)$.

Keywords

- scribes

- efficiency

- electronic health record In a retrospective analysis of 20 clinic sessions and 438 patients 12 to 18 months after intervention, session time increased to $266.0 \pm 22.0$ minutes on average, but the average number of patients per session increased to $21.9 \pm 2.8$ minutes.

Conclusion Utilizing scribes in an ophthalmology practice can increase efficiency, allowing more patients to be seen or allowing time for other activities such as teaching or research.
\end{abstract}

Electronic health records (EHR) have been associated with advantages in clinical practice such as increased patient safety, enhanced retrieval and maintenance of patients' record, decreased medication errors, and better communication between providers, ${ }^{1-4}$ all of which improve clinical care. However, the EHR has posed certain challenges such as high implementation cost, initial training requirement, reduction in clinical volume, ${ }^{3,5}$ concerns of negative patient-physician received

January 20, 2020

accepted after revision

September 22, 2020
DOI https://doi.org/

10.1055/s-0040-1721066. ISSN 2475-4757.
Copyright $\odot 2020$ by Thieme Medical Publishers, Inc., 333 Seventh Avenue, New York, NY 10001, USA. Tel: +1(212) 760-0888.
License terms

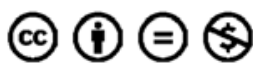


relationship, and increased physician's documentation. ${ }^{1,2,4}$ The latter is associated with increased work burden, physician dissatisfaction, and burnout. ${ }^{6}$ Sinsky et al reported that for every hour a physician has direct contact with patients, an additional 2 hours are spent on EHRs and administrative tasks in clinic. Another 1 to 2 hours each night outside office hours were spent for clerical work. $^{7}$

In ophthalmology, the addition of the EHR has generated a variety of opinions, but it is widely known that utilizing EHRs in practice requires a significant amount of time for physicians. ${ }^{5,8}$ One study reports that the EHR accounted for $27 \%$ of ophthalmologist's time during patient encounters, using 10.8 minutes on average per encounter and 1.9 hours per half-day clinic session. ${ }^{9}$ The burden of the EHR on ophthalmologists specifically has been shown in several studies to have a negative impact on productivity, with an increase in documentation time but little to no increase or even reduction in clinical volume. , $^{5,10}$

Scribes have been shown to alleviate this clerical burden and reduce physician burnout. Gidwani et al demonstrated that scribes improved charting efficiency and physician satisfaction with no detrimental effect on patient satisfaction in a family medicine clinic in an academic center. ${ }^{2}$ In addition, productivity, revenue, and patient-physician interaction were shown to improve with the use of scribes. ${ }^{4,11-13}$ However, there have been no published studies to date evaluating the effect of incorporating scribes into ophthalmology practice. The aim of this study is to determine what effect scribes have on efficiency in an academic ophthalmology clinic.

\section{Methods}

This is a quality improvement study conducted by two attending ophthalmologists at UT Health San Antonio from January 2018 to April 2018. Implementation of medical scribes was the primary intervention. The main objectives of the scribes were to record exam findings as dictated by the physician, provide in-room support to the physician, and facilitate patient flow through clinic. In-room support varied with the type of patient encounter but would consist of reviewing medication regimens, cleaning equipment, or retrieving additional supplies. This study assigned scribe duties to individuals who were previously trained as ophthalmic technicians, thus having knowledge of the field and terminology. Clinic session time, patient encounter time, number of patients seen per session, and template time adherence were recorded before and after intervention. Session time was defined as start time of physician with the first patient until the last patient encounter was completed. Clinic sessions typically represented a half-day morning or afternoon, excluding extended break periods. These data points were recorded by the scribe on a data sheet during each encounter, which prevented masking them to the study. Template time was measured as the time allotted in a physician's schedule for a particular encounter type such as preoperative follow-up, procedure, or new patient evaluation. Unpaired two-tailed Student $t$-test and statistical process control charts were used for statistical analysis.
A second component of our analysis included a retrospective study of session time and number of patients seen per session 12 to 18 months after implementation of scribes into practice to evaluate whether this intervention allowed the provider to increase patient load. Clinic sessions 12 to 18 months after the intervention were chosen at random for evaluation. Session time was again defined as start time of physician with first patient until last patient in clinic session and determined based on time stamps in EHR. All statistical analyses were completed using QI Macros and Microsoft Excel.

\section{Results}

Eighty-three patients and 17 clinic sessions were included in the preintervention dataset; 169 patients and 21 clinic sessions were included the post-intervention dataset. The average number of patients per session was kept similar preand post-intervention at $15.8 \pm 4.4$ and $14.9 \pm 2.8$, respectively $(p=0.45)$. Mean preintervention session time was $265.0 \pm 31.4$ minutes, in contrast to $223.4 \pm 19.9$ minutes post-intervention $(p<0.001)$ as seen in - Fig. $\mathbf{1}$. The session time data from each clinic session was trended over the 4month period and is depicted in - Fig. 2. Mean preintervention patient encounter time was $15.0 \pm 8.3$ minutes, while the mean encounter time post-intervention was $10.9 \pm 7.0$ minutes $(p<0.005)$ as seen in - Fig. 3 . Template time adherence improved from $73 \%$ preintervention to $89 \%$ post-intervention $(p<0.001)$. In the second component of our study, the retrospective analysis of 20 clinic sessions with total of 438 patients was completed to determine the long-term effects of scribes on practice efficiency 12 to 18 months post-intervention. Overall, mean session time utilizing scribes for one ophthalmologist increased to $266.0 \pm 22.0$ minutes. However, the number of patients seen per session increased from $14.9 \pm 2.8$ to $21.9 \pm 2.8$ over the 12 to 18 months period post-intervention. Based on this data, physicians spent an average of 12.1 minutes per patient per session time.

\section{Discussion}

To our knowledge, this is the first study evaluating the use of medical scribes in an ophthalmology practice and their effect on efficiency. Our study found that using scribes in an ophthalmology practice improved clinical efficiency, demonstrated by a reduction in mean clinic session time and mean patient encounter time. For our practice, this created additional time to increase patient volume in future clinic sessions, demonstrated by the increase in patients seen per session 12 to 18 months post-intervention. The key findings in our report are consistent with studies from multiple other specialties utilizing scribes in practice..$^{2,11-14}$ Our study also identifies specific actions for scribes to carry out that can increase physician and practice efficiency. These actions include facilitating patient flow through clinic and providing in-room support to physicians, in addition to helping with the electronic medical record. These steps increased efficiency in each patient encounter and allowed for comprehensive, 


\section{Session Time per Clinic Session}

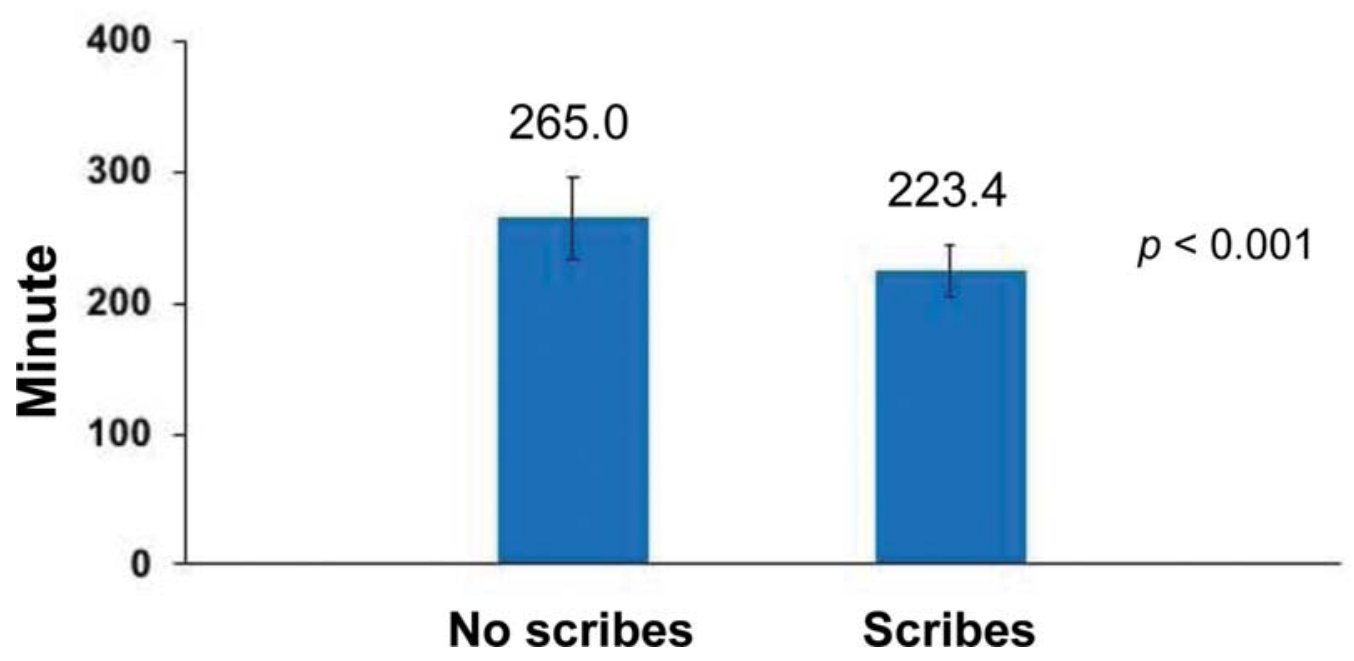

Fig. 1 Session time per clinic session.

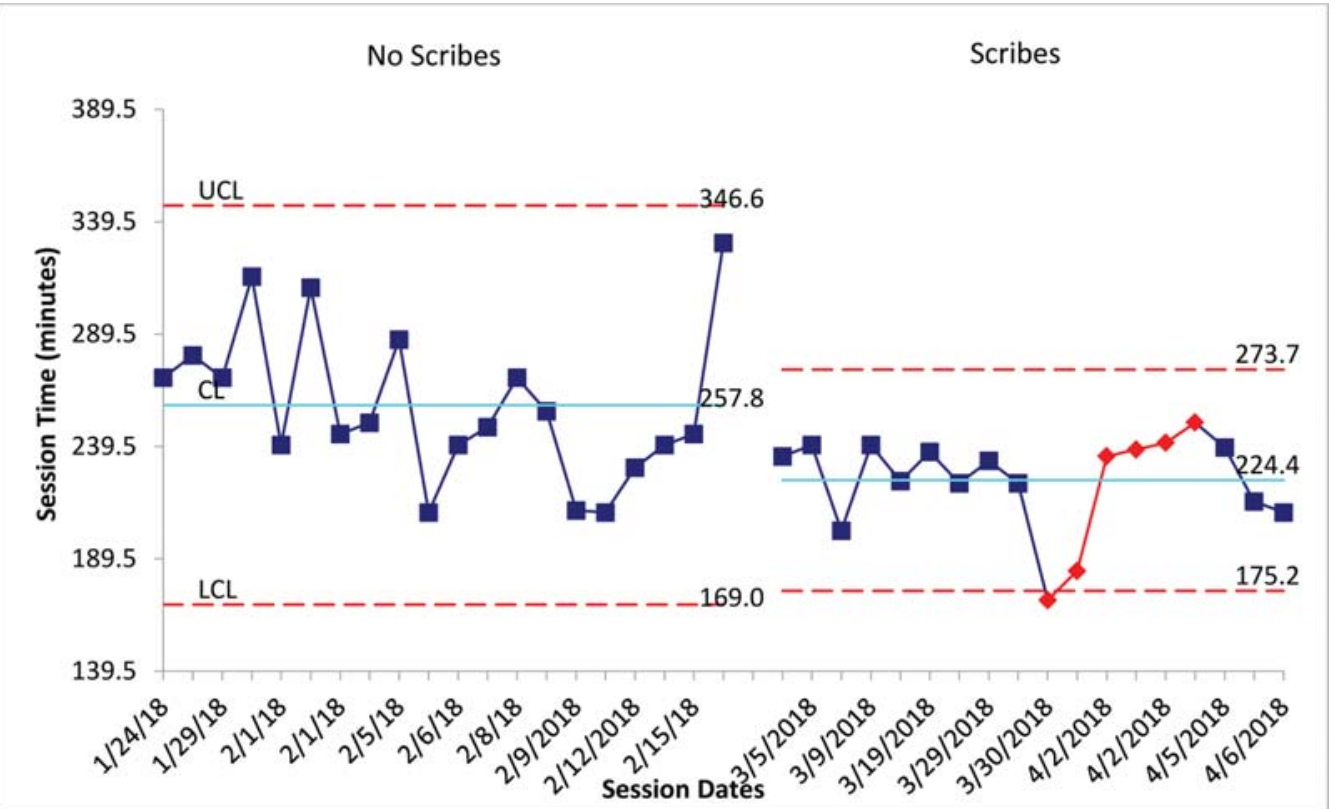

Fig. 2 Statistical process control chart for session time before and after scribe implementation. Key: UCL, upper control limit; LCL, lower control limit.

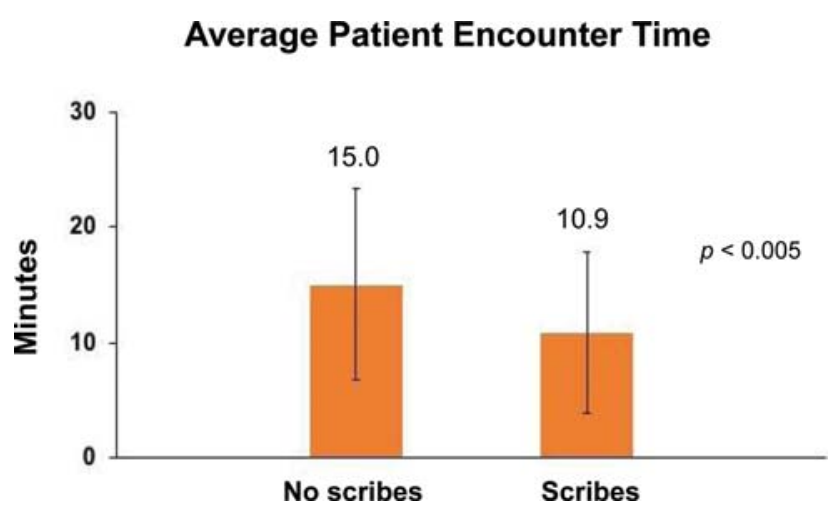

Fig. 3 Average patient encounter time. personalized care in shorter amount of time. Our study also emphasizes the long-term effect of scribes in practice over a 12- to 18-month period post-intervention. While the mean session time increased overall during this period, the total patient volume also increased. These data suggest that scribes allowed for increased efficiency in clinic, creating more time for additional patients and a subsequent increase in patient volume.

In addition, the decrease in clinic session time allows for a variety of options for utilization of that time in practice based on individual needs or requirements. We chose to increase the amount of patients seen per half-day session, in an effort to increase access to care based on the need in our 
population. In general, as the population begins to age, the gap between eye care providers and patients is increasing. ${ }^{15}$ Because of this finding, time and efficiency in ophthalmology has become increasingly valuable. Implementing scribes into practice could have an additive effect on improving access to care for the aging population. Other institutions and practices may utilize the time in different ways including dedicated research, quality improvement projects, or resident education.

Further, financial considerations were taken into account with regards to incorporating a scribe into practice. Given that our own ophthalmic technicians were trained as scribes, that meant an additional ophthalmic technician would need to be hired to account for the patient screenings that the scribe was no longer doing. Thus, a return on investment was calculated. Hiring a new technician costs approximately $\$ 50,000$ annually. The average reimbursement for an office visit is approximately $\$ 148$ (internal departmental data). We took into account approximately 247 days spent in clinic each year when subtracting weekends and holidays. Thus, adding two patients each day would equate to $\$ 73,112$ additional per year. This would result in an approximate return on investment of $\$ 23,112$ per year to the provider. Thus, adding a scribe seems makes financial sense as well as improving efficiency and access to care.

Some limitations to the study should be noted. This study was limited to only two physicians in the same geographic region and practice type, and thus findings might not be generalizable to different areas or specialties. Our implementation of scribe duties was performed by previously trained ophthalmic technicians. Traditional implementation of newly-hired scribes might require additional time for training, familiarization with space, and the EHR and could affect the speed of noticeable change in practice. Our practice also utilizes residents working in conjunction with physicians which could have affected overall timing in some clinic sessions and should be considered as a possible confounding factor. In addition, patient and physician satisfaction were not evaluated in our study. However, many studies in other fields have noted favorable increases in physician satisfaction without changes in patient satisfaction. ${ }^{1,2,11-13}$ Also, this study did not address what effect scribes may have on patient wait times. The aforementioned limitations warrant future investigation and study.

\section{Conclusion}

Overall, our findings support increased efficiency with implementation of scribes into ophthalmology practice. Specifically, scribes reduce the mean clinic session time and mean patient encounter time. These effects allow for additional time within a workday to increase patient volume or focus on quality improvement projects, research, and resident education in an academic setting.
Conflict of Interest

None declared.

\section{Acknowledgments}

Each author listed in this publication has no financial disclosures and has not utilized personal assistance for the preparation, formatting, or publication of this data.

\section{References}

1 Pozdnyakova A, Laiteerapong N, Volerman A, et al. Impact of medical scribes on physician and patient satisfaction in primary care. J Gen Intern Med 2018;33(07):1109-1115

2 Gidwani R, Nguyen C, Kofoed A, et al. Impact of scribes on physician satisfaction, patient satisfaction, and charting efficiency: a randomized controlled trial. Ann Fam Med 2017;15(05):427-433

3 Lam JG, Lee BS, Chen PP. The effect of electronic health records adoption on patient visit volume at an academic ophthalmology department. BMC Health Serv Res 2016;16:7

4 Shultz CG, Holmstrom HL. The use of medical scribes in health care settings: a systematic review and future directions. J Am Board Fam Med 2015;28(03):371-381

5 Redd TK, Read-Brown S, Choi D, Yackel TR, Tu DC, Chiang MF. Electronic health record impact on productivity and efficiency in an academic pediatric ophthalmology practice. J AAPOS 2014;18 (06):584-589

6 Gardner RL, Cooper E, Haskell J, et al. Physician stress and burnout: the impact of health information technology. J Am Med Inform Assoc 2019;26(02):106-114

7 Sinsky C, Colligan L, Li L, et al. Allocation of physician time in ambulatory practice: a time and motion study in 4 specialties. Ann Intern Med 2016;165(11):753-760

8 Goldstein IH, Hwang T, Gowrisankaran S, Bales R, Chiang MF, Hribar MR. Changes in electronic health record use time and documentation over the course of a decade. Ophthalmology 2019;126(06):783-791

9 Read-Brown S, Hribar MR, Reznick LG, et al. Time requirements for electronic health record use in an academic ophthalmology center. JAMA Ophthalmol 2017;135(11):1250-1257

10 Chiang MF, Read-Brown S, Tu DC, et al. Evaluation of electronic health record implementation in ophthalmology at an academic medical center (an American Ophthalmological Society thesis). Trans Am Ophthalmol Soc 2013;111:70-92

11 Martel ML, Imdieke BH, Holm KM, et al. Developing a medical scribe program at an Academic Hospital: the Hennepin County Medical Center Experience. Jt Comm J Qual Patient Saf 2018;44 (05):238-249

12 Hess JJ, Wallenstein J, Ackerman JD, et al. Scribe impacts on provider experience, operations, and teaching in an academic emergency medicine practice. West J Emerg Med 2015;16(05): 602-610

13 Koshy S, Feustel PJ, Hong M, Kogan BA. Scribes in an ambulatory urology practice: patient and physician satisfaction. J Urol 2010; 184(01):258-262

14 Zallman L, Finnegan K, Roll D, Todaro M, Oneiz R, Sayah A. Impact of medical scribes in primary care on productivity, face-to-face time, and patient comfort. J Am Board Fam Med 2018;31(04): 612-619

15 Resnikoff S, Felch W, Gauthier TM, Spivey B. The number of ophthalmologists in practice and training worldwide: a growing gap despite more than 200,000 practitioners. Br J Ophthalmol 2012;96(06):783-787 